\title{
CELL DIVISION
}

\section{Repo-Man's extra exit strategy}

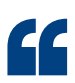

\section{Repo-Man actually has two distinct anaphase functions}

Protein phosphatase $1 \gamma(\mathrm{PP} 1 \gamma)$ is recruited to mitotic chromatin at anaphase by its regulatory subunit Repo-Man (also known as CDCA2), where it promotes dephosphorylation of histone $\mathrm{H} 3$ and chromosome decondensation. Vagnarelli et al. now confirm these results and show that Repo-Man actually has two distinct anaphase functions that are mediated by opposite ends of the protein: chromatin remodelling and coordination of nuclear envelope reformation.

The authors previously showed that Repo-Man is phosphorylated in vitro by cyclin-dependent kinase 1 (CDK1)-cyclin B. Here, they identify Thr412 as a key residue for this reaction: mutating Thr412 to Ala led to premature localization

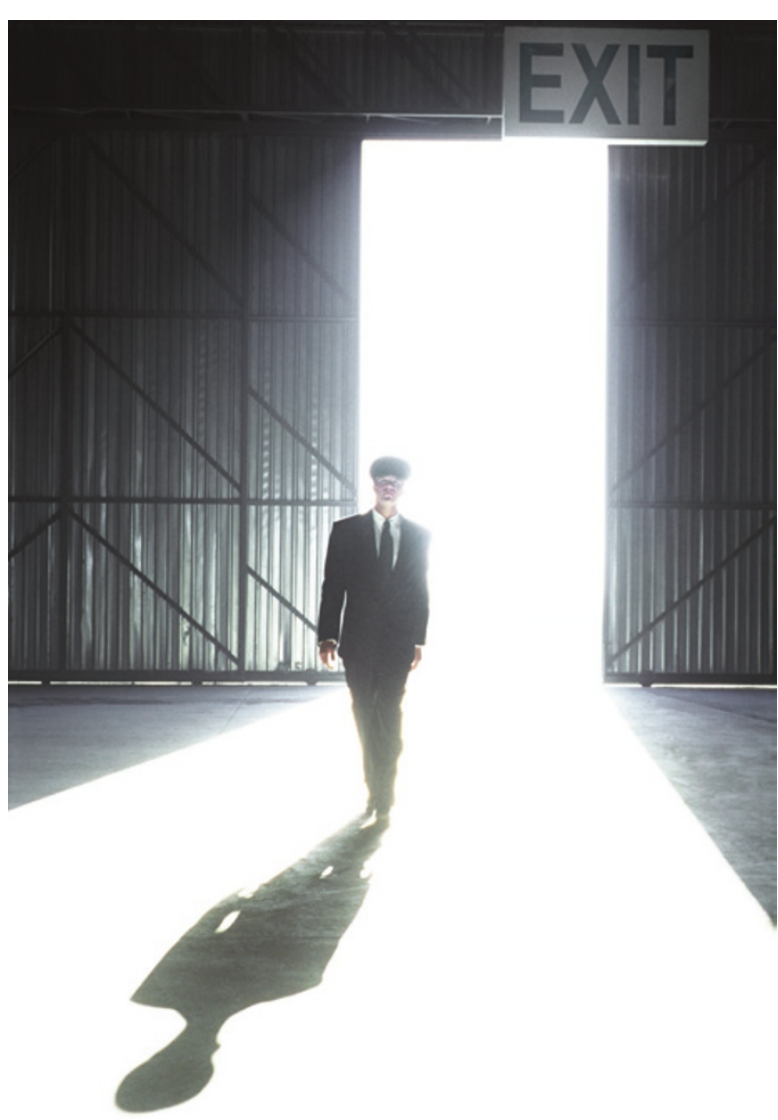

of Repo-Man to chromosomes. However, this early chromosomal localization was blocked if the PP1 $\gamma$-binding site was mutated, suggesting that $\mathrm{PP} 1 \gamma$ is also required for this localization. Therefore, they conclude that CDK1-cyclin B phosphorylates Repo-Man at multiple sites to prevent PP $1 \gamma$ binding and keep it off chromosomes until anaphase.

They then identified factors that interact with Repo-Man during mitotic exit. Pull-down experiments detected not only the expected PP1 $\gamma$ subunits and histones but also importin- $\beta$ and nucleoporin 153 , factors that are important for nuclear envelope reassembly. Repo-Man partially colocalized with importin- $\beta$ at the periphery of chromosomes in anaphase. Moreover, importin- $\beta$ bound directly to an aminoterminal domain of Repo-Man (residues 1-135). This interaction was specific to anaphase, independent of the PP1 $\gamma$-binding site and negatively controlled by CDK-mediated phosphorylation of Repo-Man.

The idea that Repo-Man might regulate not only chromosome organization but also early steps in nuclear envelope reassembly at the chromosome periphery was supported by analysis of its dynamic localization. A carboxy-terminal (403-1023) fragment of Repo-Man localized to bulk chromatin rapidly after anaphase but was not at the periphery of chromosomes, whereas an N-terminal (1-397) region accumulated exclusively at the periphery a few minutes later.
Moreover, Repo-Man was required for both normal chromatin reorganization in G1 and nuclear reassembly. RNA interferencemediated depletion of Repo-Man interfered with normal histone $\mathrm{H} 3$ dephosphorylation and reduced heterochromatin protein $1 \alpha$ accumulation on chromatin and subsequent assembly of heterochromatin foci during mitotic exit. It also resulted in irregular nuclear morphology, which the authors attributed to defective importin- $\beta$ localization.

The authors propose that the N-terminal section of Repo-Man is important for organizing components needed early on for nuclear reassembly, whereas the C-terminal section is required for PP1 $\gamma$-mediated modification of histone $\mathrm{H} 3$ to promote chromatin remodelling. Further work is needed to determine the mechanisms by which the $\mathrm{N}$-terminal and C-terminal domains of Repo-Man control its different anaphase functions. However, this study highlights the importance of having a common regulator coordinating chromosome condensation and nuclear envelope reassembly.

Antony F. Bickenson

ORIGINAL RESEARCH PAPER Vagnarelli, P. et al. Repo-Man coordinates chromosomal reorganization with nuclear envelope reassembly during mitotic exit. Dev. Cell 21, 328-342 (2011) FURTHER READING Qian, J. et al. PP1/Repo-Man dephosphorylates mitotic histone $\mathrm{H} 3$ at $\mathrm{T} 3$ and regulates chromosomal Aurora B targeting. Curr. Biol. 21, 766-773 (2011)| Wurzenberger, C. \& Gerlich, D. W. Phosphatases: providing safe passage through mitotic exit. Nature Rev. Mol. Cell Biol. 12, 469-482 (2011) 